\title{
Effect of Rotation on Double-Diffusive Convection in a Magnetized Ferrofluid with Internal Angular Momentum
}

\author{
Sunil $^{1 \dagger}$, P. Chand ${ }^{2}$, A. Mahajan ${ }^{3}$ and P. Sharma ${ }^{1}$ \\ ${ }^{1}$ Department of Mathematics, National Institute of Technology, Hamirpur, H.P. -177 005, India \\ ${ }^{2}$ Department of Applied Sciences, Jawahar Lal Nehru Government Engineering College, Sunder Nagar, \\ H.P. -174 401, India \\ ${ }^{3}$ Department of Mathematics, Central University of Himachal Pradesh, Dharamshala, - 176 215, India
}

$\dagger$ Corresponding Author Email: sunilnitham@gmail.com

(Received June 22, 2009; accepted June 21, 2010)

\begin{abstract}
This paper deals with the theoretical investigation of the effect of rotation in a magnetized ferrofluid with internal angular momentum, heated and soluted from below subjected to a transverse uniform magnetic field. For a flat fluid layer contained between two free boundaries, an exact solution is obtained. A linear stability analysis theory and normal mode analysis method have been carried out to study the onset of convection. The influence of various parameters like rotation, solute gradient, magnetization and internal angular momentum parameters (i.e. coupling parameter, spin diffusion parameter and heat conduction parameter) has been analyzed on the onset of stationary convection. The critical magnetic thermal Rayleigh number for the onset of instability is also determined numerically for sufficiently large values of buoyancy magnetization parameter $M_{1}$ and results are depicted graphically. The principle of exchange of stabilities is found to hold true for the ferrofluid with internal angular momentum heated from below in the absence of rotation, coupling between vorticity and spin, microinertia and solute gradient. The oscillatory modes are introduced due to the presence of the rotation, coupling between vorticity and spin, microinertia and solute gradient, which were non-existent in their absence. In this paper, an attempt is also made to obtain the sufficient conditions for the non-existence of overstability.
\end{abstract}

Keywords: Ferrofluid, Double-diffusive convection, Rotation, Internal angular momentum, Magnetization.

\section{INTRODUCTION}

Magnetic fluids or ferrofluids are colloidal suspensions of fine ferromagnetic mono domain nanoparticles in non-conducting liquids. The ferromagnetic nanoparticles are coated with a surfactant to prevent their agglomeration. Rosensweig $(1985,1987)$ in his monograph and review article, provides a detailed introduction to this subject. Chandrasekhar (1981) has given a detailed account of thermal convection problems of Newtonian fluids. The theory of convective instability of ferrofluid begins with Finlayson (1970) and interestingly continued by Lalas and Carmi (1971), Shliomis (1974), Stiles and Kagan (1990), Venkatasubramanian and Kaloni (1994) and Sunil and co-workers (2005, 2008). Schwab et al. (1983) investigated experimentally the Finlayson's problem in the case of a strong magnetic field and detected the onset of convection by plotting the Nusselt number versus the Rayleigh number. Then, the critical Rayleigh number corresponds to a discontinuity in the slope. Later, Stiles and Kagan (1990) examined the experimental problem reported by Schwab et al. (1983) and generalized the Finlayson's model assuming that under a strong magnetic field, the rotational viscosity augments the shear viscosity. In the absence of applied magnetic field, the particles in the colloidal suspensions are randomly oriented and thus the fluid has no net magnetization. When exposed to a magnetic field, Brownian rotational motions prevent complete alignment of the dipoles with the applied field. As a result when the applied field has a changing direction or magnitude, the magnetization is unable to track the field closely and becomes non-equilibrated. This nonequilibrium state of magnetization leads to the state of asymmetric stress. Rayleigh-Bénard convection in a ferromagnetic fluid layer with internal angular momentum permeated by a uniform, vertical magnetic field with free-free, isothermal, spin-vanishing, magnetic boundaries has been considered by Abraham (2002). She observed that the micropolar ferromagnetic fluid layer heated from below is more stable as compared with the classical Newtonian ferromagnetic fluid. More recently, Sunil et al. (2004) have studied the convection problems in ferrofluid with internal angular momentum. 
The really interesting situation from both a geophysical and a mathematical viewpoint arises when the layer is simultaneously heated from below and salted from below. In the standard Bénard problem, the instability is driven by a density difference caused by a temperature difference between the upper and lower planes bounding the fluid. If the fluid layer additionally has salt dissolved in it then there are potentially two destabilizing sources for the density difference, the temperature field and the salt field. When there are two effects such as this, the phenomenon of convection which arises is called double-diffusive convection. For the specific case involving a temperature field and sodium chloride, it is frequently referred to as thermohaline convection. There are many recent studies involving three or more fields, such as temperature and two salts such as $\mathrm{NaCl}, \mathrm{KCl}$. For three or greater field case, it is referred to as multi-component convection. The driving force for many studies in double-diffusive or multi-component convection is largely physical applications. Sunil et al. (2004) have studied the thermosolutal convection in a ferrofluid and Sunil et al. (2007a, b) studied the double-diffusive convection in a micropolar ferromagnetic fluid in non-porous and porous media.

In view of the above investigations, we intend to extend our work to the double-diffusive convection in ferrofluid with internal angular momentum in the presence of rotation. It is attempted to discuss the influence of rotation and solute gradient and how the angular momentum parameters affects the stability in ferrofluid heated from below. The understanding of the rotating ferrofluid stability problems plays an important role in microgravity environmental applications. We believe that the present study can serve as the theoretical support for experimental investigation in ferroconvection. This problem, to the best of our knowledge, has not been investigated yet.

\section{Mathematical Formulation OF THE ProbleM}

Here, we consider an infinite horizontal layer of thickness ' $d$ ' of an electrically non-conducting incompressible thin rotating ferrofluid with internal angular momentum heated and soluted from below as shown in Fig. 1. The temperature $\mathrm{T}$ and solute concentration $\mathrm{C}$ at the bottom and top surfaces $z=\mp \frac{1}{2} d$ are $T_{L}, \mathrm{~T}_{U}$ and $C_{L}, \mathrm{C}_{U}$, respectively, and a uniform temperature gradient $\beta\left(=\left|\frac{d T}{d z}\right|\right)$ and a uniform solute gradient $\beta^{\prime}\left(=\left|\frac{d C}{d z}\right|\right)$ are maintained.

Here, both the boundaries are taken to be free and perfect conductors of heat. The gravity field $\mathbf{g}=(0,0,-g)$ and uniform vertical magnetic field intensity $\mathbf{H}=\left(0,0, H_{0}\right)$ pervade the system. The whole system is assumed to be rotating with angular velocity $\boldsymbol{\Omega}=(0,0, \Omega)$ along the vertical axis, which is taken as z-axis.

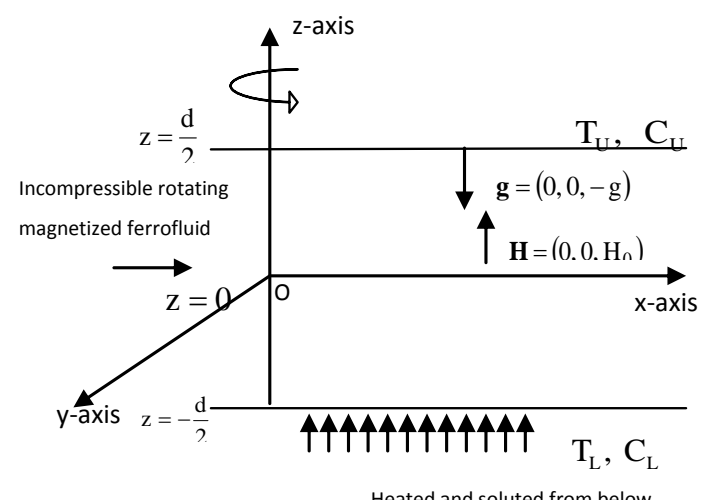

Fig. 1. Geometrical configuration

The mathematical equations governing the motion of magnetized ferrofluids for the above model (Rosensweig 1985; Chandrasekhar 1981; Finlayson 1970; Sunil et al. 2007b) are as follows:

The continuity equation for an incompressible fluid is

$\nabla \cdot \mathbf{q}=0$.

The momentum and internal angular momentum equations are

$$
\begin{aligned}
& \rho_{0}\left(\frac{\partial}{\partial t}+\mathbf{q} \cdot \nabla\right) \mathbf{q}=-\nabla p+\rho \mathbf{g}+\mu_{0}(\mathbf{M} \cdot \nabla) \mathbf{H}+(\zeta+\eta) \nabla^{2} \mathbf{q} \\
&+ 2 \zeta(\nabla \times \boldsymbol{\omega})+2 \rho_{0}(\mathbf{q} \times \mathbf{\Omega}), \\
& \rho_{0} I\left[\frac{\partial}{\partial t}+(\mathbf{q} \cdot \nabla)\right] \boldsymbol{\omega}=2 \zeta(\nabla \times \mathbf{q}-2 \boldsymbol{\omega})+\mu_{0}(\mathbf{M} \times \mathbf{H}) \\
&+\left(\lambda^{\prime}+\eta^{\prime}\right) \nabla(\nabla \cdot \boldsymbol{\omega})+\eta^{\prime} \nabla^{2} \boldsymbol{\omega} .
\end{aligned}
$$

The temperature and solute concentration equations for an incompressible ferrofluid are (Finlayson (1970), Abraham (2002), Sunil et al. (2004, 2007a, b))

$$
\begin{gathered}
{\left[\rho_{0} C_{V, H}-\mu_{0} \mathbf{H} \cdot\left(\frac{\partial \mathbf{M}}{\partial T}\right)_{V, \mathbf{H}}\right] \frac{D T}{D t}+\mu_{0} T\left(\frac{\partial \mathbf{M}}{\partial T}\right)_{V, \mathbf{H}} \cdot \frac{D \mathbf{H}}{D t}} \\
=K_{1} \nabla^{2} T+\delta(\nabla \times \boldsymbol{\omega}) \cdot \nabla T \\
{\left[\rho_{0} C_{V, H}-\mu_{0} \mathbf{H} \cdot\left(\frac{\partial \mathbf{M}}{\partial C}\right)_{V, \mathbf{H}}\right] \frac{D C}{D t}} \\
+\mu_{0} C\left(\frac{\partial \mathbf{M}}{\partial C}\right)_{V, \mathbf{H}} \cdot \frac{D \mathbf{H}}{D t}=K_{1}^{\prime} \nabla^{2} C
\end{gathered}
$$

The density equation of state is

$$
\rho=\rho_{0}\left[1-\alpha\left(T-T_{a}\right)+\alpha^{\prime}\left(C-C_{a}\right)\right],
$$

where $\rho, \rho_{0}, \mathbf{q}, \boldsymbol{\omega}, \mathrm{t}, \mathrm{p}, \eta, \zeta, \lambda^{\prime}, \eta^{\prime}, \delta, I, \mu_{0}$, $\mathbf{B}, C_{V, \mathrm{H}}, \mathbf{M}, K_{1}, K_{1}^{\prime}, \alpha$ and $\alpha^{\prime}$ are the fluid density, reference density, velocity, microrotation, time, pressure, shear kinematic viscosity coefficient, coupling viscosity coefficient or vortex viscosity, bulk spin viscosity coefficient, shear spin viscosity coefficient, heat conduction coefficient, moment of inertia (microinertia constant), magnetic permeability of free 
space $\left(\mu_{0}=4 \pi \times 10^{-7}\right.$ Henry $\left.\mathrm{m}^{-1}\right), \quad$ magnetic induction, heat capacity at constant volume and magnetic field, magnetization, thermal conductivity, solute conductivity, thermal expansion coefficient and an analogous solvent coefficient of expansion, respectively. $T_{a}$ is the average temperature given by $T_{a}=\frac{\left(T_{L}+T_{U}\right)}{2}$, where $T_{L}$ and $T_{U}$ are the constant average temperatures of the lower and upper surfaces of the layer and $C_{a}$ is the average concentration given by $C_{a}=\frac{\left(C_{L}+C_{U}\right)}{2}$, where $C_{L}$ and $C_{U}$ are the constant average concentrations of the lower and upper surfaces of the layer. The effect of rotation contributes two terms: (a) Centrifugal force $-\frac{1}{2} \operatorname{grad}|\boldsymbol{\Omega} \times \mathbf{r}|^{2}$ and (b) Coriolis force $2(\boldsymbol{\Omega} \times \mathbf{q})$. In Eq. $p=p_{f}-\frac{1}{2} \rho_{0}|\mathbf{\Omega} \times \mathbf{r}|^{2}$ is the reduced pressure, where $p_{f}$ stands for fluid pressure. The partial derivatives of $\mathbf{M}$ are material properties which can be evaluated once the magnetic equation of state, such as (10) below is known.

Maxwell's equations, simplified for a non-conducting fluid with no displacement currents, become

$\nabla \cdot \mathbf{B}=0, \quad \nabla \times \mathbf{H}=\mathbf{0}$,

where the magnetic induction is given by

$\mathbf{B}=\mu_{0}(\mathbf{H}+\mathbf{M})$.

In general, the presence of ferrofluid can distort an external magnetic field if magnetic interaction (dipoledipole) takes place, but this is negligible for small particle concentrations, as is assumed here. The magnetization depends on the magnitude of the magnetic field, temperature and salinity, which can be written as

$$
\mathbf{M}=\frac{\mathbf{H}}{H} M(H, \mathrm{~T}, \mathrm{C}) .
$$

The magnetic equation of state is linearized about the magnetic field, $H_{0}$, an average temperature, $T_{a}$, and an average concentration, $C_{a}$ to become

$$
M=M_{0}+\chi\left(H-H_{0}\right)-K_{2}\left(T-T_{a}\right)+K_{3}\left(C-C_{a}\right),
$$

where magnetic susceptibility, pyromagnetic coefficient and salinity magnetic coefficient are defined by

$$
\begin{aligned}
& \chi \equiv\left(\frac{\partial M}{\partial H}\right)_{H_{0}, \mathrm{~T}_{\mathrm{a}}, \mathrm{C}_{\mathrm{a}}}, \quad K_{2} \equiv-\left(\frac{\partial M}{\partial T}\right)_{H_{0}, \mathrm{~T}_{\mathrm{a}}, \mathrm{C}_{\mathrm{a}} \text { and }} \\
& K_{3} \equiv\left(\frac{\partial M}{\partial C}\right)_{H_{0}, \mathrm{~T}_{\mathrm{a}}, \mathrm{C}_{\mathrm{a}}}, \text { respectively. }
\end{aligned}
$$

Here $H_{0}$ is the uniform magnetic field of the fluid layer when placed in an external magnetic field
$\mathbf{H}=H_{0}^{e x t} \hat{\mathbf{k}}, \hat{\mathbf{k}}$ is unit vector in the z-direction, $M_{0}=M\left(H_{0}, T_{a}, \mathrm{C}_{\mathrm{a}}\right)$.

The basic state is assumed to be quiescent state and is given by

$$
\begin{aligned}
& \mathbf{q}=\mathbf{q}_{b}=\left(\begin{array}{ll}
0, & 0,0
\end{array}\right), \quad \boldsymbol{\omega}=\boldsymbol{\omega}_{b}=(0,0,0), \quad \rho=\rho_{b}(z), \\
& p=p_{b}(z), T=T_{b}(z)=-\beta z+T_{a}, \quad \beta=\frac{T_{L}-T_{U}}{d}, \\
& C=C_{b}(z)=-\beta^{\prime} z+C_{a}, \mathbf{H}_{b}=\left[H_{0}-\frac{K_{2} \beta z}{1+\chi}+\frac{K_{3} \beta^{\prime} z}{1+\chi}\right] \hat{\mathbf{k}} \\
& \beta^{\prime}=\frac{C_{L}-C_{U}}{d}, \quad \mathbf{M}_{b}=\left[M_{0}+\frac{K_{2} \beta z}{1+\chi}-\frac{K_{3} \beta^{\prime} z}{1+\chi}\right] \hat{\mathbf{k}} \quad \text { and } \\
& H_{0}+M_{0}=H_{0}^{\text {ext }},
\end{aligned}
$$

where the subscript ' $b$ ' denotes the basic state.

\section{MATHEMATICAL ANALYSIS DISPERSION RELATION}

AND

We shall analyze the stability of the basic state by introducing the following perturbations:

$$
\begin{aligned}
& \mathbf{q}=\mathbf{q}_{b}+\mathbf{q}^{\prime}, \boldsymbol{\omega}=\boldsymbol{\omega}_{b}+\boldsymbol{\omega}^{\prime}, \rho=\rho_{b}+\rho^{\prime}, p=p_{b}(z)+p^{\prime}, \\
& T=T_{b}(z)+\theta, C=C_{b}(z)+\gamma, \\
& \mathbf{H}=\mathbf{H}_{b}(z)+\mathbf{H}^{\prime} \text { and } \mathbf{M}=\mathbf{M}_{b}(z)+\mathbf{M}^{\prime},
\end{aligned}
$$

where

$\mathbf{q}^{\prime}=(u, \mathrm{v}, \mathrm{w}), \boldsymbol{\omega}^{\prime}=\left(\omega_{1}, \omega_{2}, \omega_{3}\right), \rho^{\prime}, p^{\prime}, \theta, \gamma, \mathbf{H}^{\prime}$

and $\mathbf{M}^{\prime}$ are perturbations in velocity $\mathbf{q}$, spin $\boldsymbol{\omega}$, density $\rho$, pressure $\mathrm{p}$, temperature $\mathrm{T}$, concentration $\mathrm{C}$, magnetic field intensity $\mathbf{H}$ and magnetization $\mathbf{M}$, respectively.

The change in density $\rho^{\prime}$, caused mainly by the perturbations $\theta$ and $\gamma$ in temperature and concentration, respectively, is given by

$$
\rho^{\prime}=-\rho_{0}\left(\alpha \theta-\alpha^{\prime} \gamma\right) \text {. }
$$

Then, the linearized perturbation equations of the magnetized ferrofluid become

$$
\begin{aligned}
& \rho_{0} \frac{\partial u}{\partial t}=-\frac{\partial p^{\prime}}{\partial x}+\mu_{0}\left(M_{0}+H_{0}\right) \frac{\partial H_{1}^{\prime}}{\partial z}+(\zeta+\eta) \nabla^{2} u \\
& +2 \zeta \Omega_{1}^{\prime}+2 \rho_{0} \Omega v, \\
& \rho_{0} \frac{\partial v}{\partial t}=-\frac{\partial p^{\prime}}{\partial y}+\mu_{0}\left(M_{0}+H_{0}\right) \frac{\partial H_{2}^{\prime}}{\partial z}+(\zeta+\eta) \nabla^{2} v \\
& +2 \zeta \Omega_{2}^{\prime}-2 \rho_{0} \Omega u, \\
& \rho_{0} \frac{\partial w}{\partial t}=-\frac{\partial p^{\prime}}{\partial z}+\mu_{0}\left(M_{0}+H_{0}\right) \frac{\partial H_{3}^{\prime}}{\partial z}+(\zeta+\eta) \nabla^{2} w \\
& +2 \zeta \Omega_{3}^{\prime}-\frac{\mu_{0} K_{2} \beta}{1+\chi}\left\{H_{3}^{\prime}(1+\chi)-K_{2} \theta\right\}+g \rho_{0}\left(\alpha \theta-\alpha^{\prime} \gamma\right) \\
& +\frac{\mu_{0} K_{3} \beta^{\prime}}{1+\chi}\left\{H_{3}^{\prime}(1+\chi)+K_{3} \gamma\right\}-\frac{\mu_{0} K_{2} K_{3}}{(1+\chi)}\left(\beta^{\prime} \theta+\beta \gamma\right) \text {, }
\end{aligned}
$$


Sunil et al. / JAFM, Vol. 4, No. 4, pp. 43-52, 2011.

$$
\begin{aligned}
\rho_{0} I \frac{\partial \boldsymbol{\omega}^{\prime}}{\partial t}= & 2 \zeta\left(\nabla \times \mathbf{q}^{\prime}-2 \boldsymbol{\omega}^{\prime}\right)+\mu_{0}\left(\mathbf{M}_{b} \times \mathbf{H}^{\prime}+\mathbf{M}^{\prime} \times \mathbf{H}_{b}\right) \\
& +\left(\lambda^{\prime}+\eta^{\prime}\right) \nabla\left(\nabla \cdot \boldsymbol{\omega}^{\prime}\right)+\eta^{\prime} \nabla^{2} \boldsymbol{\omega}^{\prime} \\
\frac{\partial u}{\partial x}+\frac{\partial v}{\partial y}+ & \frac{\partial w}{\partial z}=0 \\
\rho C_{1} \frac{\partial \theta}{\partial t}- & \mu_{0} T_{0} K_{2} \frac{\partial}{\partial t}\left(\frac{\partial \Phi_{1}^{\prime}}{\partial z}\right)=K_{1} \nabla^{2} \theta \\
& +\left[\rho C_{1} \beta-\frac{\mu_{0} T_{0} K_{2}^{2} \beta}{(1+\chi)}\right] w-\delta \beta \Omega_{3}^{\prime} \\
\rho C_{1}^{\prime} \frac{\partial \gamma}{\partial t}- & \mu_{0} C_{0} K_{3} \frac{\partial}{\partial t}\left(\frac{\partial \Phi_{2}^{\prime}}{\partial z}\right)=K_{1}^{\prime} \nabla^{2} \gamma \\
& +\left[\rho C_{1}^{\prime} \beta^{\prime}-\frac{\mu_{0} C_{0} K_{3}^{2} \beta^{\prime}}{(1+\chi)}\right] w \\
(1+\chi) \frac{\partial^{2} \Phi^{\prime}}{\partial z^{2}} & +\left(1+\frac{M_{0}}{H_{0}}\right) \nabla_{1}^{2} \Phi^{\prime}-K_{2} \frac{\partial \theta}{\partial z}+K_{3} \frac{\partial \gamma}{\partial z}=0
\end{aligned}
$$

where $\rho C_{1}=\rho_{0} C_{V, H}+\mu_{0} K_{2} H_{0}$,

$\rho C_{1}^{\prime}=\rho_{0} C_{V, H}-\mu_{0} K_{3} H_{0}, \quad \mathbf{H}^{\prime}=\nabla \Phi^{\prime}=\nabla\left(\Phi_{1}^{\prime}-\Phi_{2}^{\prime}\right)$ (by Eq. (7)) where $\Phi^{\prime}$ is the perturbed magnetic potential which is the difference of two potentials $\Phi_{1}^{\prime}, \Phi_{2}^{\prime}$ analogous to temperature and solute.

Eliminating $\mathrm{u}, \mathrm{v}, p^{\prime}$ between Eqs. (14)-(16), using Eq. (18), and taking curl once on (17) and considering only z-component, we obtain

$$
\begin{aligned}
\left(\rho_{0} \frac{\partial}{\partial t}-(\right. & \left.\zeta+\eta) \nabla^{2}\right) \nabla^{2} w= \\
& -\frac{\mu_{0} K_{2} \beta}{1+\chi} \nabla_{1}^{2}\left\{(1+\chi) \frac{\partial}{\partial z}\left(\Phi_{1}^{\prime}-\Phi_{2}^{\prime}\right)-K_{2} \theta\right\} \\
& +\frac{\mu_{0} K_{3} \beta^{\prime}}{1+\chi} \nabla_{1}^{2}\left\{(1+\chi) \frac{\partial}{\partial z}\left(\Phi_{1}^{\prime}-\Phi_{2}^{\prime}\right)+K_{3} \gamma\right\} \\
& +\rho_{0} g \nabla_{1}^{2}\left(\alpha \theta-\alpha^{\prime} \gamma\right)-2 \rho_{0} \Omega \frac{\partial \zeta_{1}}{\partial z} \\
& -\frac{\mu_{0} K_{2} K_{3}}{(1+\chi)} \nabla_{1}^{2}\left(\beta^{\prime} \theta+\beta \gamma\right)+2 \zeta \nabla^{2} \Omega_{3}^{\prime}, \\
\rho_{0} I \frac{\partial \Omega_{3}^{\prime}}{\partial t}= & -2 \zeta\left(\nabla^{2} w+2 \Omega_{3}^{\prime}\right)+\eta^{\prime} \nabla^{2} \Omega_{3}^{\prime} .
\end{aligned}
$$

The vertical component of the vorticity equation is

$\rho_{0} \frac{\partial \zeta_{1}}{\partial t}=2 \rho_{0} \Omega \frac{\partial w}{\partial z}+(\zeta+\eta) \nabla^{2} \zeta_{1}$,

where $\zeta_{1}=\frac{\partial v}{\partial x}-\frac{\partial u}{\partial y}$ stands for the z-component of the vorticity.

Further analysis has been carried out using the techniques of Sunil et al. (2007b, 2008).

Now we analyze the normal mode technique. This can be written as

$$
f(x, y, z, t)=f(z, t) \exp i\left(k_{x} x+k_{y} y\right),
$$

where $f(z, t)$ represents $W(z, t), \Theta(z, t), Z(z, t)$, $\Gamma(z, t), \Phi_{1}(z, t), \Phi_{2}(z, t), \Omega_{3}(z, t) ; k_{x}, k_{y}$ are the wave numbers along the $x^{-}$and $y$-directions, respectively and $k=\sqrt{\left(k_{x}^{2}+k_{y}^{2}\right)}$ is the resultant wave number.

Following the normal mode analysis, the linearized perturbation dimensionless equations become

$$
\begin{aligned}
& \left\{\frac{\partial}{\partial t^{*}}-\left(1+N_{1}\right)\left(D^{2}-a^{2}\right)\right\}\left(D^{2}-a^{2}\right) W^{*}= \\
& a R^{1 / 2}\left[\left(M_{1}-M_{4}\right) D \Phi_{1}^{*}-\left(1+M_{1}-M_{4}\right) T^{*}\right] \\
& +a S^{1 / 2}\left[\left(M_{1}^{\prime}-M_{4}^{\prime}\right) D \Phi_{2}^{*}+\left(1-M_{1}^{\prime}+M_{4}^{\prime}\right) C^{*}\right] \\
& +2 N_{1}\left(D^{2}-a^{2}\right) \Omega_{3}^{*}-T_{A}^{1 / 2} D Z^{*}, \\
& \left\{\frac{\partial}{\partial t^{*}}-\left(1+N_{1}\right)\left(D^{2}-a^{2}\right)\right\} Z^{*}=T_{A}^{1 / 2} D W^{*}, \\
& I^{\prime} \frac{\partial \Omega_{3}^{*}}{\partial t^{*}}=-2 N_{1}\left\{\left(D^{2}-a^{2}\right) W^{*}+2 \Omega_{3}^{*}\right\}+N_{3}\left(D^{2}-a^{2}\right) \Omega_{3}^{*}, \\
& P_{r} \frac{\partial T^{*}}{\partial t^{*}}-P_{r} M_{2} \frac{\partial}{\partial t^{*}}\left(D \Phi_{1}^{*}\right)=\left(D^{2}-a^{2}\right) T^{*} \\
& +a R^{1 / 2}\left(1-M_{2}\right) W^{*}-a R^{1 / 2} N_{5} \Omega_{3}^{*}, \\
& P_{s} \frac{\partial C^{*}}{\partial t^{*}}-P_{s} M_{2}^{\prime} \frac{\partial}{\partial t^{*}}\left(D \Phi_{2}^{*}\right)=\left(D^{2}-a^{2}\right) C^{*} \\
& +a S^{1 / 2}\left(1-M_{2}^{\prime}\right) W^{*}, \\
& D^{2} \Phi_{1}^{*}-a^{2} M_{3} \Phi_{1}^{*}-D T^{*}=0 \text {, } \\
& D^{2} \Phi_{2}^{*}-a^{2} M_{3} \Phi_{2}^{*}-D C^{*}=0 \text {. }
\end{aligned}
$$

where the following non-dimensional quantities and non-dimensional parameters are introduced:

$$
\begin{aligned}
& t^{*}=\frac{v t}{d^{2}}, W^{*}=\frac{W d}{v}, \Phi_{1}^{*}=\frac{(1+\chi) K_{1} a R^{1 / 2}}{K_{2} \rho C_{1} \beta v d^{2}} \Phi_{1}, \\
& \Phi_{2}^{*}=\frac{(1+\chi) K_{1}^{\prime} a S^{1 / 2}}{K_{3} \rho C_{1}^{\prime} \beta^{\prime} v d^{2}} \Phi_{2}, R=\frac{g \alpha \beta d^{4} \rho C_{1}}{v K_{1}}, \\
& S=\frac{g \alpha^{\prime} \beta^{\prime} d^{4} \rho C_{1}^{\prime}}{v K_{1}^{\prime}}, T^{*}=\frac{K_{1} a R^{1 / 2}}{\rho C_{1} \beta v d} \Theta, C^{*}=\frac{K_{1}^{\prime} a S^{1 / 2}}{\rho C_{1}^{\prime} \beta^{\prime} v d} \Gamma, \\
& a=k d, z^{*}=\frac{z}{d}, D=\frac{\partial}{\partial z^{*}}, P_{r}=\frac{v}{K_{1}} \rho C_{1}, \\
& P_{s}=\frac{v}{K_{1}^{\prime}} \rho C_{1}^{\prime}, M_{1}=\frac{\mu_{0} K_{2}^{2} \beta}{(1+\chi) \alpha \rho_{0} g}, \\
& M_{1}^{\prime}=\frac{\mu_{0} K_{3}^{2} \beta^{\prime}}{(1+\chi) \alpha^{\prime} \rho_{0} g}, M_{2}=\frac{\mu_{0} T_{0} K_{2}^{2}}{(1+\chi) \rho C_{1}}, \\
& M_{2}^{\prime}=\frac{\mu_{0} C_{0} K_{3}^{2}}{(1+\chi) \rho C_{1}^{\prime}}, M_{3}=\frac{\left(1+\frac{M_{0}}{H_{0}}\right)}{(1+\chi)}, \\
& M_{4}=\frac{\mu_{0} K_{2} K_{3} \beta^{\prime}}{(1+\chi) \alpha \rho_{0} g}, M_{4}^{\prime}=\frac{\mu_{0} K_{2} K_{3} \beta}{(1+\chi) \alpha^{\prime} \rho_{0} g},
\end{aligned}
$$


Sunil et al. / JAFM, Vol. 4, No. 4, pp. 43-52, 2011.

$M_{5}=\frac{M_{4}}{M_{1}}=\frac{M_{1}^{\prime}}{M_{4}^{\prime}}=\frac{K_{3} \beta^{\prime}}{K_{2} \beta}, N_{1}=\frac{\zeta}{\eta}, N_{3}=\frac{\eta^{\prime}}{\eta d^{2}}$,

$N_{5}=\frac{\delta}{\rho C_{1} d^{2}}, I^{\prime}=\frac{I}{d^{2}}$ and $\Omega_{3}^{*}=\frac{\Omega_{3} d^{3}}{v}$,

$T_{A}=\left(\frac{2 \Omega d^{2}}{v}\right)^{2}$

The exact solution subject to the boundary conditions (for free-free boundaries)

$W^{*}=D^{2} W^{*}=T^{*}=C^{*}=\Omega_{3}^{*}=D \Phi_{1}^{*}=D \Phi_{2}^{*}=0 \quad$ at

$z= \pm \frac{1}{2}$

is written in the form

$$
\begin{aligned}
& W^{*}=A_{1} e^{\sigma \mathrm{t}^{*}} \cos \pi z^{*}, T^{*}=B_{1} e^{\sigma \mathrm{t}^{*}} \cos \pi z^{*}, \\
& D \Phi_{1}^{*}=C_{1} e^{\sigma \mathrm{t}^{*}} \cos \pi z^{*}, \Phi_{1}^{*}=\left(\frac{C_{1}}{\pi}\right) e^{\sigma \mathrm{t}^{*}} \sin \pi z^{*}, \\
& \Omega_{3}^{*}=D_{1} e^{\sigma \mathrm{t}^{*}} \cos \pi z^{*}, D \Phi_{2}^{*}=E_{1} e^{\sigma \mathrm{t}^{*}} \cos \pi z^{*}, \\
& \Phi_{2}^{*}=\left(\frac{E_{1}}{\pi}\right) e^{\sigma \mathrm{t}^{*}} \sin \pi z^{*}, C^{*}=F_{1} e^{\sigma \mathrm{t}^{*}} \cos \pi z^{*},
\end{aligned}
$$

where $A_{1}, \mathrm{~B}_{1}, C_{1}, \mathrm{D}_{1}, \mathrm{E}_{1}, \mathrm{~F}_{1}$, are constants and $\sigma$ is the growth rate which is, in general, a complex constant.

Substituting solution (34) in linearized perturbation dimensionless equations and dropping asterisks for convenience, we get the equations involving the coefficients of $A_{1}, \mathrm{~B}_{1}, C_{1}, \mathrm{D}_{1}, \mathrm{E}_{1}, \mathrm{~F}_{1}$. For the existence of non-trivial solutions, the determinant of the coefficients of $A_{1}, \mathrm{~B}_{1}, C_{1}, \mathrm{D}_{1}, \mathrm{E}_{1}, \mathrm{~F}_{1}$ must vanish. This determinant on simplification yields

$$
i T_{5} \sigma_{i}^{5}+T_{4} \sigma_{i}^{4}-i T_{3} \sigma_{i}^{3}-T_{2} \sigma_{i}^{2}+i T_{1} \sigma_{i}+T_{0}=0 .
$$

Here,

$$
\begin{aligned}
T_{5}= & b I_{1} L_{2} L_{2}^{\prime}, \\
T_{4}= & b^{2}\left\{L_{1} I_{1}\left(L_{2}+L_{2}^{\prime}\right)+N_{3}^{\prime} L_{2} L_{2}^{\prime}\right\} \\
& +2 b L_{2} L_{2}^{\prime}\left\{\left(1+N_{1}\right) I_{1}+2 N_{1}\right\}
\end{aligned}
$$

$$
\begin{aligned}
+ & \left(4 N_{1}+b N_{3}^{\prime}\right)\left\{x S_{1}\left(1-M_{2}^{\prime}\right) L_{2} L_{3}^{\prime}-x R_{1}\left(1-M_{2}\right) L_{2}^{\prime} L_{3}\right\} \\
& +b\left\{2 x R_{1} N_{1} N_{5}^{\prime} L_{2}^{\prime} L_{3}+T_{A_{1}} I_{1} L_{1}\left(L_{2}+L_{2}^{\prime}\right)\right\} \\
+ & b\left\{-x R_{1}\left(1-M_{2}\right) I_{1} L_{1} L_{3}+x S_{1}\left(1-M_{2}^{\prime}\right) I_{1} L_{1} L_{3}^{\prime}\right\},(39) \\
T_{1}= & -4 L_{1}^{2} N_{1}^{2} b^{4}+b^{4} L_{1}\left(1+N_{1}\right)\left\{\begin{array}{l}
L_{1} I_{1} b\left(1+N_{1}\right) \\
-4 N_{1}^{2}\left(L_{2}+L_{2}^{\prime}\right)
\end{array}\right\} \\
+ & b^{2}\left(1+N_{1}\right)\left\{2 N_{1} x R_{1} N_{5}^{\prime} L_{2}^{\prime} L_{3}-L_{1} x R_{1}\left(1-M_{2}\right) I_{1} L_{3}\right\} \\
& +b^{2}\left(1+N_{1}\right) L_{1} x S_{1}\left(1-M_{2}^{\prime}\right) I_{1} L_{3}^{\prime} \\
& +b\left(1+N_{1}\right)\left[L_{1} b^{3}\left(4 N_{1}+b N_{3}^{\prime}\right)\left(1+N_{1}\right)\left(L_{2}+L_{2}^{\prime}\right)\right] \\
+ & b\left(1+N_{1}\right)\left[x\left\{S_{1}\left(1-M_{2}^{\prime}\right) L_{2} L_{3}^{\prime}-R_{1}\left(1-M_{2}\right) L_{2}^{\prime} L_{3}\right\}\right] \\
+ & \left(4 N_{1}+b N_{3}^{\prime}\right) b L_{1}\left\{x S_{1}\left(1-M_{2}^{\prime}\right) L_{3}^{\prime}-x R_{1}\left(1-M_{2}\right) L_{3}\right\} \\
+ & \left(4 N_{1}+b N_{3}^{\prime}\right) b L_{1}\left\{T_{A_{1}}\left(L_{2}+L_{2}^{\prime}\right)+2 L_{1} b^{3}\left(1+N_{1}\right)\right\} \\
+ & b^{2} L_{1}\left\{2 N_{1} x R_{1} N_{5}^{\prime} L_{3}+L_{1} I_{1} T_{A_{1}}\right\}, \\
T_{0}= & -x R_{1}\left(1-M_{2}\right) L_{3}\left(4 N_{1}+b N_{3}^{\prime}\right) b^{2} L_{1}\left(1+N_{1}\right) \\
+ & \left(4 N_{1}+b N_{3}^{\prime}\right) b^{2} L_{1}\left(1+N_{1}\right)\left[x S_{1}\left(1-M_{2}^{\prime}\right) L_{3}^{\prime}+L_{1} b^{3}\left(1+N_{1}\right)\right] \\
+ & 2 b^{3} L_{1} N_{1}\left(1+N_{1}\right)\left\{x R_{1} N_{5}^{\prime} L_{3}-2 L_{1} N_{1} b^{2}\right\} \\
& +\left(4 N_{1}+b N_{3}^{\prime}\right) L_{1}^{2} b^{2} T_{A_{1}},
\end{aligned}
$$

where

$R_{1}=\frac{R}{\pi^{4}}, S_{1}=\frac{S}{\pi^{4}}, x=\frac{a^{2}}{\pi^{2}}, \mathrm{i} \sigma_{\mathrm{i}}=\frac{\sigma}{\pi^{2}}$,

$\mathrm{I}_{1}=\pi^{2} \mathrm{I}^{\prime}, \mathrm{N}_{3}^{\prime}=\pi^{2} \mathrm{~N}_{3}, \mathrm{~N}_{5}^{\prime}=\pi^{2} \mathrm{~N}_{5}, T_{A_{1}}=\frac{T_{A}}{\pi^{4}}$,

$b=1+x, L_{1}=\left(1+x M_{3}\right), L_{2}=P_{r}\left[1-M_{2}+x M_{3}\right]$,

$L_{2}^{\prime}=P_{s}\left[1-M_{2}^{\prime}+x M_{3}\right]$,

$L_{3}=\left[1+x M_{3}+x M_{3} M_{1}\left(1-M_{5}\right)\right]$ and

$L_{3}^{\prime}=\left[1+x M_{3}+x M_{3} M_{1}^{\prime}\left(\frac{1}{M_{5}}-1\right)\right]$.

\section{Results ANd Discussion}

\subsection{The Case of Stationary Convection}

When the instability sets in as stationary convection (and $M_{2} \cong 0, \quad M_{2}^{\prime} \cong 0$ ), the marginal state will be characterized by $\sigma_{i}=0$, then the Rayleigh number is given by

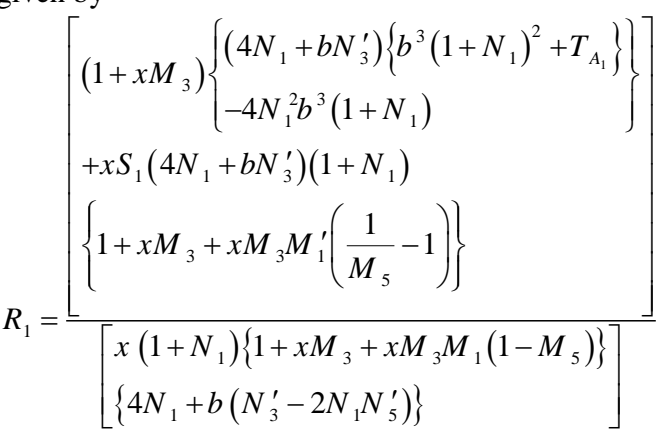


which expresses the modified Rayleigh number $R_{1}$ as a function of the dimensionless wave number $\mathrm{x}$, buoyancy magnetization parameter $M_{1}$ (ratio of magnetic to gravitational forces), magnetization parameter $M_{3}$ (measures the departure of linearity in the magnetic equation of state), Taylor number $T_{A_{1}}$, solute gradient parameter $S_{1}$, ratio of the salinity effect on magnetic field to pyromagnetic coefficient $M_{5}$, coupling parameter $N_{1}$ (coupling between vorticity and spin effects), spin diffusion parameter $N_{3}^{\prime}$ and heat conduction parameter $N_{5}^{\prime}$ (coupling between spin and heat fluxes).

The classical results in respect of Newtonian fluids can be obtained as the limiting case of present study.

Setting $N_{1}=0$ and $S_{1}=0$, and keeping $N_{3}^{\prime}$ arbitrary in Eq. (42), we get

$$
R_{1}=\frac{\left(1+x M_{3}\right)\left\{(1+x)^{3}+T_{A_{1}}\right\}}{x\left\{1+x M_{3}\left(1+M_{1}\right)\right\}},
$$

which is the expression for Rayleigh number of ferrofluid in the presence of rotation.

Setting $M_{3}=0$ in Eq. (43), we get

$$
R_{1}=\frac{(1+x)^{3}+T_{A_{1}}}{x},
$$

In the absence of rotation $T_{A_{1}}$ it further reduces to

$$
R_{1}=\frac{(1+x)^{3}}{x}
$$

the classical Rayleigh Bénard result for the Newtonian fluid case.

To investigate the effect of rotation, solute gradient, magnetization parameter, coupling parameter, spin diffusion parameter and heat conduction parameter, we examine the behavior of

$\frac{d R_{1}}{d T_{A_{1}}}, \frac{d R_{1}}{d S_{1}}, \frac{d R_{1}}{d M_{3}}, \frac{d R_{1}}{d N_{1}}, \frac{d R_{1}}{d N_{3}^{\prime}}$ and $\frac{d R_{1}}{d N_{5}^{\prime}}$

analytically.

Eq. (42) gives

$$
\begin{aligned}
& \frac{d R_{1}}{d T_{A_{1}}}=\frac{L_{1}\left(4 N_{1}+b N_{3}^{\prime}\right)}{x L_{3}\left(1+N_{1}\right)\left[4 N_{1}+b\left(N_{3}^{\prime}-2 N_{1} N_{5}^{\prime}\right)\right]}, \\
& \frac{d R_{1}}{d S_{1}}=\frac{\left(4 N_{1}+b N_{3}^{\prime}\right)\left\{1+x M_{3}+x M_{3} M_{1}^{\prime}\left(\frac{1}{M_{5}}-1\right)\right\}}{L_{3}\left[4 N_{1}+b\left(N_{3}^{\prime}-2 N_{1} N_{5}^{\prime}\right)\right]} .
\end{aligned}
$$

This shows that, rotation and solute gradient have a stabilizing role if $N_{3}^{\prime}>2 N_{1} N_{5}^{\prime}$. In the absence of coupling parameter $N_{1}$; rotation and solute gradient always have a stabilizing effect on the onset of convection.

Eq. (42) also yields:

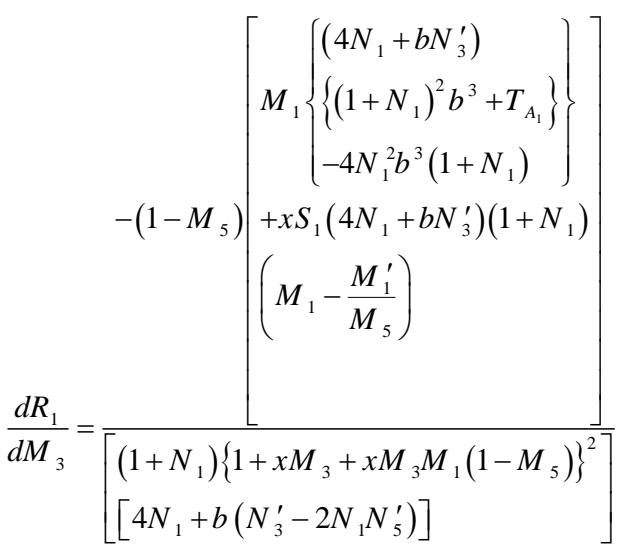

which is always negative if $N_{3}^{\prime}>2 N_{1} N_{5}^{\prime}$ and $M_{1} M_{5}>M_{1}^{\prime}$.

This shows that the magnetization parameter has a destabilizing effect when conditions (49) hold. In the absence of coupling parameter $\left(N_{1}=0\right)$ and the effect on magnetization due to salinity $\left(M_{1}^{\prime}=0\right)$, the magnetization parameter always has a destabilizing effect on the system.

It follows from Eq. (42) that

$$
\begin{aligned}
& L_{1}\left[4 N_{1}+b\left(N_{3}^{\prime}-2 N_{1} N_{5}^{\prime}\right)\right] \\
& {\left[\begin{array}{l}
4 b^{3}\left(1+N_{1}\right)^{2}+4 T_{A_{1}} \\
+b N_{3}^{\prime}\left\{b^{3}\left(1+N_{1}\right)^{2}-T_{A_{1}}\right\}
\end{array}\right] } \\
& +4 x b S_{1} L_{3}^{\prime}\left(1+N_{1}\right)^{2}\left[4 N_{1}+b\left(N_{3}^{\prime}-2 N_{1} N_{5}^{\prime}\right)\right] \\
& +2\left(1+N_{1}\right)\left(b N_{5}^{\prime}-2\right) \\
\frac{d R_{1}}{d N_{1}}= & {\left[\begin{array}{l}
\left.4 L_{1}\left\{\begin{array}{l}
4 N_{1} b^{3}\left(1+N_{1}\right)+4 N_{1} T_{A_{1}} \\
+b N_{3}^{\prime}\left\{\left(1+N_{1}\right)^{2} b^{3}+T_{A_{1}}\right\}
\end{array}\right\}\right] \\
+x b S_{1} L_{3}^{\prime}\left(4 N_{1}+b N_{3}^{\prime}\right)\left(1+N_{1}\right)
\end{array}\right] }
\end{aligned}
$$

which is always positive if $N_{3}^{\prime}>2 N_{1} N_{5}^{\prime}, T_{A_{1}}<1$ and $N_{5}^{\prime}>2$.

This shows that coupling parameter has a stabilizing effect when conditions (51) hold.

Eq. (42) gives

$$
\frac{d R_{1}}{d N_{3}^{\prime}}=-\frac{2 b N_{1}\left[\begin{array}{l}
N_{5}^{\prime}\left\{b\left(1+x M_{3}\right) T_{A_{1}}+x S_{1}\left(1+N_{1}\right) L_{3}^{\prime}\right\} \\
+b^{2} L_{1}\left(1+N_{1}\right)\left\{b^{2} N_{5}^{\prime}\left(1+N_{1}\right)-2 N_{1}\right\}
\end{array}\right]}{\left[\begin{array}{l}
x\left(1+N_{1}\right)\left\{1+x M_{3}+x M_{3} M_{1}\left(1-M_{5}\right)\right\} \\
{\left[4 N_{1}+b\left(N_{3}^{\prime}-2 N_{1} N_{5}^{\prime}\right)\right]^{2}}
\end{array}\right]},
$$

which is always negative if $N_{5}^{\prime}>2$.

This shows that the spin diffusion parameter has a destabilizing effect when condition (53) holds. 
Eq. (42) also yields

$$
\frac{d R_{1}}{d N_{5}^{\prime}}=\frac{\left[\begin{array}{l}
L_{1}\left\{\begin{array}{l}
\left(4 N_{1}+N_{3}^{\prime} b\right)\left\{\left(1+N_{1}\right)^{2} b^{3}+T_{A_{1}}\right\} \\
-4 N_{1}^{2} b^{3}\left(1+N_{1}\right)
\end{array}\right\} \\
+x S_{1}\left(4 N_{1}+b N_{3}^{\prime}\right)\left(1+N_{1}\right) \\
\left\{1+x M_{3}+x M_{3} M_{1}^{\prime}\left(\frac{1}{M_{5}}-1\right)\right\}
\end{array}\right]}{x L_{3}\left(1+N_{1}\right)\left[4 N_{1}+b\left(N_{3}^{\prime}-2 N_{1} N_{5}^{\prime}\right)\right]^{2}},
$$

which is always positive. This shows that the heat conduction always has a stabilizing effect.

For sufficiently large values of $M_{1}$ (Finlayson 1970), we obtain the results for the magnetic mechanism

$$
R_{m}=\frac{\left[\begin{array}{l}
\left(1+x M_{3}\right)\left\{\begin{array}{l}
\left(4 N_{1}+b N_{3}^{\prime}\right)\left\{b^{3}\left(1+N_{1}\right)^{2}+T_{A_{1}}\right\} \\
-4 N_{1}^{2} b^{3}\left(1+N_{1}\right)
\end{array}\right\} \\
+x S_{1}\left(4 N_{1}+b N_{3}^{\prime}\right)\left(1+N_{1}\right) \\
\left\{1+x M_{3}+x M_{3} M_{1}^{\prime}\left(\frac{1}{M_{5}}-1\right)\right\}
\end{array}\right]}{x^{2} M_{3}\left(1-M_{5}\right)\left(1+N_{1}\right)\left\{4 N_{1}+b\left(N_{3}^{\prime}-2 N_{1} N_{5}^{\prime}\right)\right\}},
$$

where $R_{m}$ is the magnetic thermal Rayleigh number.

As a function of $x, R_{m}$ given by Eq. (55) attains its minimum when

$$
P_{6} x^{6}+P_{5} x^{5}+P_{4} x^{4}+P_{3} x^{3}+P_{2} x^{2}+P_{1} x+P_{0}=0 .
$$

The coefficients $P_{0}, \ldots \ldots, P_{6}$, being quite lengthy, has not been written here and are evaluated during numerical calculations.

The values of critical wave number for the onset of instability are determined numerically using NewtonRaphson method by the condition $\frac{d R_{m}}{d x}=0$. With $x$ determined as a solution of Eq. (56), Eq. (55) will give the required critical magnetic thermal Rayleigh number $N_{c}$ which depends on $M_{3}, T_{A_{1}}, S_{1}, N_{1}, \mathrm{~N}_{3}^{\prime}$ and $N_{5}^{\prime}$. Values of $N_{c}$ determined for various values of $M_{3}, T_{A_{1}}, S_{1}, N_{1}, \mathrm{~N}_{3}^{\prime}$ and $N_{5}^{\prime}$ are illustrated in Figs. 2-7.

Figure 2 represents the plot of critical magnetic thermal Rayleigh number $N_{c}$ versus $M_{3}$ in the presence and absence of coupling parameter $N_{1}$. This figure indicates that non-buoyancy magnetization have a destabilizing effect. Also, it is observed from the Fig. 2 that even only for small values of $N_{1}$, onset of convection is delayed. This shows that higher values of $N_{c}$ are needed for onset of convection in the presence of $N_{1}$, hence justifying the stabilizing effect of coupling parameter.

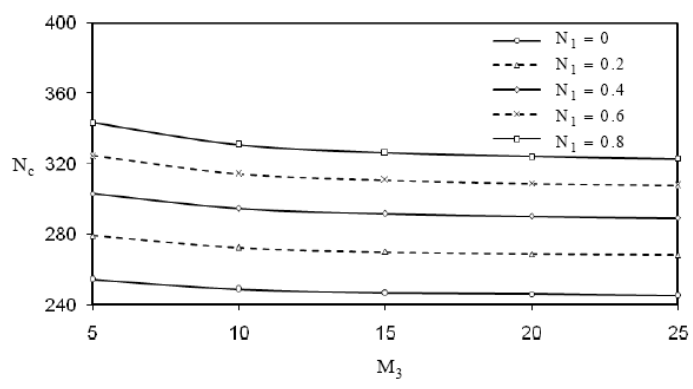

Fig. 2. Marginal instability curve for variation of $N_{c}$ versus $\mathrm{M}_{3}$ for $\mathrm{N}_{3}^{\prime}=2, \mathrm{~N}_{5}^{\prime}=0.5, \mathrm{M}_{1}^{\prime}=0.1, \mathrm{M}_{5}^{\prime}=0.2$, $\mathrm{T}_{\mathrm{A}_{1}}=100$ and $\mathrm{S}_{1}=100$.

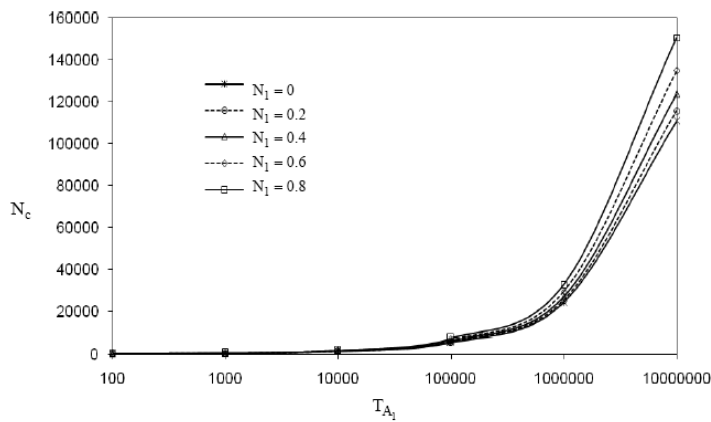

Fig. 3. Marginal instability curve for variation of $N_{c}$ versus $\mathrm{T}_{\mathrm{A}_{1}}$ for $\mathrm{M}_{3}=5, \mathrm{~N}_{3}^{\prime}=2, \mathrm{~N}_{5}^{\prime}=0.5, \mathrm{M}_{1}^{\prime}=0.1$, $\mathrm{M}_{5}^{\prime}=0.2$ and $\mathrm{S}_{1}=100$.

Figure 3 represents plot of $N_{c}$ versus $T_{A_{1}}$ in the presence and absence of coupling parameter $N_{1}$. This figure shows that rotation always has a stabilizing effect on the system. Figures 4-6 represent the plots of critical magnetic thermal Rayleigh number $N_{c}$ versus $N_{1}, N_{3}^{\prime}$ and $N_{5}^{\prime}$.

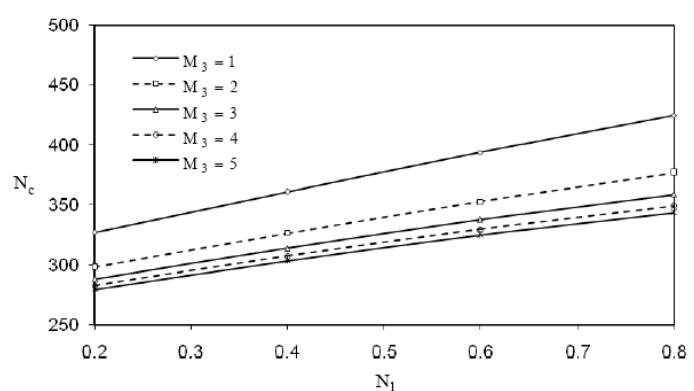

Fig. 4. Marginal instability curve for variation of $N$

versus $\mathrm{N}_{1}$ for $\mathrm{N}_{3}^{\prime}=2, \mathrm{~N}_{5}^{\prime}=0.5, \mathrm{M}_{1}^{\prime}=0.1, \mathrm{M}_{5}^{\prime}=0.2$,

$$
\mathrm{T}_{\mathrm{A}_{1}}=100 \text { and } \mathrm{S}_{1}=100 \text {. }
$$

Figures 4 and 6 indicate that the coupling parameter and heat conduction parameter has a stabilizing effect, whereas Fig. 5 indicates that the spin diffusion (couple stress) parameter have a destabilizing effect on the system. It is observed from the Fig. 4 that $N_{c}$ increases with increasing $N_{1}$. As $N_{1}$ increases, concentration of microelements also increases, and as a result of this a greater part of the energy of the system is consumed by these elements in developing twist velocities in the 
fluid, and onset of convection is delayed. In Fig. 5, we observe that $N_{c}$ decreases with increasing $N_{3}^{\prime}$. As $N_{3}^{\prime}$ increases, the couple stress of the fluid increases, which causes the microrotation to decrease; rendering the system prone to instability. Nevertheless, the above phenomenon is true in porous or non-porous medium.

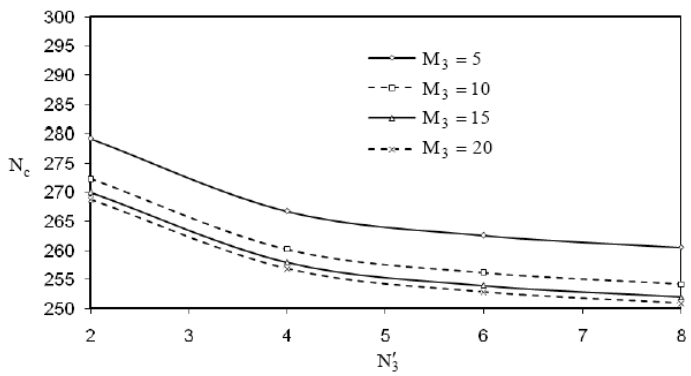

Fig. 5. Marginal instability curve for variation of $N_{c}$ versus $\mathrm{N}_{3}^{\prime}$ for $\mathrm{N}_{1}=0.2, \mathrm{~N}_{5}^{\prime}=0.5, \mathrm{M}_{1}^{\prime}=0.1, \mathrm{M}_{5}^{\prime}=0.2$,

$$
\mathrm{T}_{\mathrm{A}_{1}}=100 \text { and } \mathrm{S}_{1}=100 \text {. }
$$

Figure 6 indicates that when $N_{5}^{\prime}$ increases the heat induced into the fluid due to microelements is also increased, thus inducing the heat transfer from the bottom to the top. The decrease in heat transfer is responsible for delaying the onset of convection. Thus, increasing $N_{5}^{\prime}$ leads to an increase in $N_{c}$. In other words, $N_{5}^{\prime}$ stabilizes the flow.

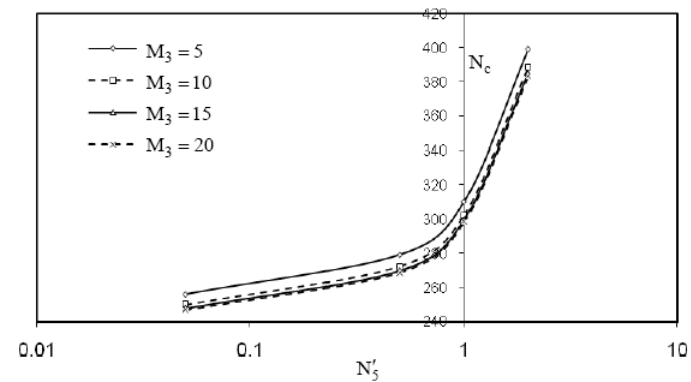

Fig. 6. Marginal instability curve for variation of $N_{c}$ versus $\mathrm{N}_{5}^{\prime}$ for $\mathrm{N}_{1}=0.2, \mathrm{~N}_{3}^{\prime}=2, \mathrm{M}_{1}^{\prime}=0.1, \mathrm{M}_{5}^{\prime}=0.2$,

$$
\mathrm{T}_{\mathrm{A}_{1}}=100 \text { and } \mathrm{S}_{1}=100 \text {. }
$$

Figure 7 represents plot of $N_{c}$ versus $S_{1}$ in the presence and absence of coupling parameter $N_{1}$. This figure indicates that the solute gradient always has a stabilizing effect when the system is soluted from below.

\subsection{The Case of Oscillatory Modes}

Here, we examine the possibility of oscillatory modes, if any, on stability problem due to the presence of magnetic parameters, angular momentum parameters, rotation and solute gradient. Equating the imaginary parts of Eq. (35), we obtain

$$
\sigma_{i}\left[T_{5} \sigma_{i}^{4}-T_{3} \sigma_{i}^{2}+T_{1}\right]=0 .
$$

It is evident from Eq. (57) that $\sigma_{i}$ may be either zero or non-zero, meaning that the modes may be either nonoscillatory or oscillatory.

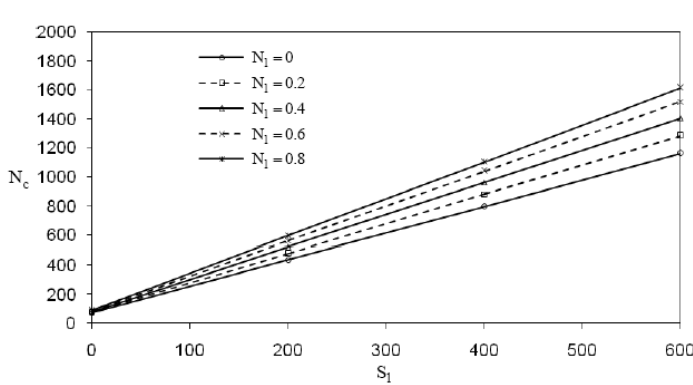

Fig. 7. Marginal instability curve for variation of $N_{c}$ versus $\mathrm{S}_{1}$ for $\mathrm{M}_{3}=5, \mathrm{~N}_{3}^{\prime}=2, \mathrm{~N}_{5}^{\prime}=0.5, \mathrm{M}_{1}^{\prime}=0.1, \mathrm{M}_{5}^{\prime}=0.2$ and $\mathrm{T}_{\mathrm{A}_{1}}=100$.

\section{Limiting case:}

In the absence of rotation $\left(T_{A_{1}}=0\right)$, the vanishing of the determinant of the coefficients of $A_{1}, \mathrm{~B}_{1}, C_{1}, D_{1}$, $E_{1}$ and $F_{1}$ obtained by substituting solution (34) in linearized perturbation dimensionless equations, gives

$T_{4} \sigma_{i}^{4}-i T_{3} \sigma_{i}^{3}-T_{2} \sigma_{i}^{2}+i T_{1} \sigma_{i}+T_{0}=0$,

Where $T_{4}=b L_{2} L_{2}^{\prime} I_{1}$,

$$
\begin{aligned}
& T_{3}=b\left[b L_{1} I_{1}\left(L_{2}+L_{2}^{\prime}\right)+\left\{\left(1+N_{1}\right) b I_{1}+4 N_{1}+b N_{3}^{\prime}\right\} L_{2} L_{2}^{\prime}\right] \text {, } \\
& T_{2}=b^{2}\left[\begin{array}{r}
L_{1}\left[b L_{1} I_{1}+\left\{\left(1+N_{1}\right) b I_{1}+4 N_{1}+b N_{3}^{\prime}\right\}\left(L_{2}+L_{2}^{\prime}\right)\right] \\
+\left\{\left(1+N_{1}\right)\left(4 N_{1}+b N_{3}^{\prime}\right)-4 N_{1}^{2}\right\} L_{2} L_{2}^{\prime}
\end{array}\right] \\
& -x R_{1}\left(1-M_{2}\right) L_{2}^{\prime} L_{3} I_{1}+x S_{1}\left(1-M_{2}^{\prime}\right) L_{2} L_{3}^{\prime} I_{1} \text {, } \\
& T_{1}=b^{3} L_{1}\left[\begin{array}{l}
\left\{\left(1+N_{1}\right)\left(4 N_{1}+b N_{3}^{\prime}\right)-4 N_{1}^{2}\right\}\left(L_{2}+L_{2}^{\prime}\right) \\
+L_{1}\left\{\left(1+N_{1}\right) b I_{1}+4 N_{1}+b N_{3}^{\prime}\right\}
\end{array}\right] \\
& -x R_{1}\left[\begin{array}{l}
\left\{\left(1-M_{2}\right)\left(4 N_{1}+b N_{3}^{\prime}\right)-2 N_{1} N_{5}^{\prime} b\right\} L_{2}^{\prime} \\
+b\left(1-M_{2}\right) L_{1} I_{1}
\end{array}\right] L_{3}, \\
& +x S_{1}\left(1-M_{2}^{\prime}\right)\left[\left(4 N_{1}+b N_{3}^{\prime}\right) L_{2}+b L_{1} I_{1}\right] L_{3}^{\prime} \text {, } \\
& T_{0}=b L_{1}\left[\begin{array}{l}
b^{3} L_{1}\left\{\left(1+N_{1}\right)\left(4 N_{1}+b N_{3}^{\prime}\right)-4 N_{1}^{2}\right\} \\
-x R_{1}\left\{\left(1-M_{2}\right)\left(4 N_{1}+b N_{3}^{\prime}\right)-2 N_{1} N_{5}^{\prime} b\right\} L_{3} \\
+x S_{1} L_{3}^{\prime}\left(1-M_{2}^{\prime}\right)\left(4 N_{1}+b N_{3}^{\prime}\right)
\end{array}\right] .
\end{aligned}
$$

Equating imaginary part of Eq. (58), we obtain

$$
\sigma_{i}\left[\begin{array}{l}
-b\left\{\begin{array}{l}
b L_{1}\left(L_{2}+L_{2}^{\prime}\right) I_{1}+ \\
L_{2} L_{2}^{\prime}\left(\left(1+N_{1}\right) b I_{1}+4 N_{1}+b N_{3}^{\prime}\right)
\end{array}\right\} \sigma_{1}^{2} \\
+b^{3} L_{1}\left\{\begin{array}{l}
L_{1}\left(\left(1+N_{1}\right) b I_{1}+4 N_{1}+b N_{3}^{\prime}\right) \\
+\left(L_{2}+L_{2}^{\prime}\right)\left(\left(1+N_{1}\right)\left(4 N_{1}+b N_{3}^{\prime}\right)-4 N_{1}^{2}\right)
\end{array}\right\} \\
-x R_{1}\left[\begin{array}{l}
\left\{\left(1-M_{2}\right)\left(4 N_{1}+b N_{3}^{\prime}\right)-2 N_{1} N_{5}^{\prime} b\right\} L_{2}^{\prime} \\
+b\left(1-M_{2}\right) L_{1} I_{1}
\end{array}\right] L_{3} \\
+x S_{1}\left(1-M_{2}^{\prime}\right)\left[\left(4 N_{1}+b N_{3}^{\prime}\right) L_{2}+b L_{1} I_{1}\right] L_{3}^{\prime}
\end{array}\right]=0
$$

It is evident from Eq. (59) that $\sigma_{i}$ may be either zero or non-zero, meaning that the modes may be either nonoscillatory or oscillatory. In the absence of viscous 
effect $\left(N_{1}=0\right)$, microinertia $\left(I_{1}=0\right)$ and solute gradient $\left(S_{1}=0\right.$ and $\left.\mathrm{L}_{2}^{\prime}=0\right)$, we obtain the result as

$\sigma_{i}\left[L_{1}+L_{2}\right]=0$.

Here the quantity inside the bracket is positive definite because the typical values of $M_{2}$ are $+10^{-6}$ (Finlayson 1970). Hence

$\sigma_{i}=0$,

which implies that the oscillatory modes are not allowed and the principle of exchange of stabilities is satisfied for ferrofluid heated from below, in the absence of viscous effect (coupling between vorticity and spin), microinertia and solute gradient. Thus, from Eq. (59), we conclude that the oscillatory modes are introduced due to the presence of the viscous effect, microinertia and solute gradient, which were nonexistent in their absence. Finally, in addition to above parameters, rotation may produce oscillatory modes.

\subsection{Sufficient Conditions for the Non-Existence of Overstability}

The present section is devoted to find the possibility that the observed instability may really be overstability. Since, we wish to determine the Rayleigh number for the onset of instability through a state of pure oscillations; it suffices to find conditions for which (35) will admit solutions with $\sigma_{i}$ real. Equating real and imaginary parts of (35) and eliminating $R_{1}$ between them, we obtain

$A_{3} c_{1}^{3}+A_{2} c_{1}^{2}+A_{1} c_{1}+A_{0}=0$,

where $A_{0}, A_{1}, A_{2}, A_{3}$ being quite lengthy has not been written here. Since $\sigma_{i}$ is real for overstability, the three values of $c_{1}\left(=\sigma_{i}^{2}\right)$ are positive. The product of roots of (62) is $-\frac{A_{0}}{A_{3}}$, and if this is to be negative, then $A_{3}>0$ and $A_{0}>0$. Now, the product is negative if

$b^{2}>T_{A_{1}}, N_{3}^{\prime} L_{2}>L_{1} I_{1}, N_{3}^{\prime} L_{2}^{\prime}>L_{1} I_{1}$,

$N_{3}^{\prime}\left(1-M_{2}\right)>4 N_{1} N_{5}^{\prime}$,

$N_{3}^{\prime}\left(1-M_{2}\right)\left(L_{2}-L_{2}^{\prime}\right)>2 L_{2} N_{1} N_{5}^{\prime}, L_{1}>N_{1} L_{2}, L_{2}>L_{1}$ and $L_{2}>L_{2}^{\prime}$,

which implies that

$T_{A_{1}}<b^{2}, N_{3}^{\prime}>\max \left\{\frac{4 N_{1} N_{5}^{\prime}}{\left(1-M_{2}\right)}, \frac{I_{1}\left(1+x M_{3}\right)}{P_{s}\left(1-M_{2}^{\prime}\right)}\right\}$,

$\frac{1}{N_{1}}>P_{r}, P_{r}>P_{s}$ and $P_{r}>\left\{\frac{P_{r} M_{2}}{1+x M_{3}}+1\right\}$.

Thus, for $\frac{1}{N_{1}}>P_{r}>\max \left\{P_{s}, 1+\frac{P_{r} M_{2}}{1+x M_{3}}\right\}$,

$N_{3}^{\prime}>\max \left\{\frac{4 N_{1} N_{5}^{\prime}}{\left(1-M_{2}\right)}, \frac{I_{1}\left(1+x M_{3}\right)}{P_{S}\left(1-M_{2}\right)}\right\}$ and $T_{A_{1}}<b^{2}$ overstability cannot occur and the principle of the exchange of stabilities is valid. Hence, the above conditions are the sufficient conditions for the nonexistence of overstability, the violation of which does not necessarily imply the occurrence of overstability.

\section{Conclusions}

The linear stability of double-diffusive convection in a magnetized rotating ferrofluid with internal angular momentum heated and soluted from below has been considered. The analysis is restricted to physical situation in which the magnetization induced by temperature and concentration variations is small compared to that induced by the external magnetic field. In conclusion, we see that convection can encourage in a ferrofluid with internal angular momentum by means of spatial variation in magnetization, which is induced when the magnetization of the fluid depends on temperature and solute concentration and a uniform temperature gradient and a uniform solute gradient are established across the layer. This problem represents thermal-salinitymicrorotational-mechanical interaction arising through the stress tensor, salinity and micro-rotation. We have investigated the effect of various parameters like magnetization, rotation, solute gradient, coupling parameter, spin diffusion parameter and heat conduction parameter on the onset of convection. The stabilizing behaviour of rotation, solute gradient are virtually unaffected by magnetic parameters but are significantly affected by angular momentum parameters. The presence of coupling between vorticity and spin effects (viscous effect), microinertia, solute gradient and rotation may bring overstability in the system.

Thus, from the above analysis, we conclude that the angular momentum parameters, rotation and solute gradient have a profound influence on the onset of convection.

\section{ACKNOWLEDGEMENTS}

The financial assistance to Dr. Sunil in the form of Research and Development Project [No. 25(0165)/08/EMR-II] of the Council of Scientific and Industrial Research (CSIR), New Delhi is gratefully acknowledged.

\section{REFERENCES}

Abraham, A. (2002). Rayleigh-Bénard convection in a micropolar ferromagnetic fluid. Int. J. Eng. Sci. 40, 449-460.

Chandrasekhar, S. (1981). Hydrodynamic and Hydromagnetic Stability. New York, Dover.

Finlayson, B.A. (1970). Convective instability of ferromagnetic fluids. J. Fluid Mech. 40, 753-767.

Lalas, D.P. and S. Carmi (1971). Thermoconvective stability of ferrofluids. Phys. Fluids 14, 436-437.

Rosensweig, R.E. (1985). Ferrohydrodynamics. Cambridge University Press, Cambridge. 
Sunil et al. / JAFM, Vol. 4, No. 4, pp. 43-52, 2011.

Rosensweig, R.E. (1987). Magnetic fluids. Rev. Fluid Mech. 19, 437-463.

Schwab, L., U. Hildebrandt and K. Stierstadt (1983). Magnetic Benardconvection. J. Magn. Magn. Mater. 39, 113-114.

Shliomis, M.I. (1974). Magnetic fluids. Sov. Phys. Usp. 17, 153-169.

Stiles, P.J. and M. Kagan (1990). Thermoconvective instability of a horizontal layer of ferrofluid in a strong vertical magnetic field. J. Magn. Magn. Mater. 85, 196-198.

Sunil, A. Sharma, Bharti and R.G. Shandil (2007a). Linear stability of double-diffusive convection in a micropolar ferromagnetic fluid saturating a porous medium. Int. J. Mech. Sci. 49, 1047-1059.

Sunil, D. Sharma and V. Sharma (2005). Effect of dust particles on rotating ferromagnetic fluid heated from below saturating a porous medium. J. Coll. Inter. Sci. 291, 152-161.

Sunil, P. Chand and P.K. Bharti (2007b). Doublediffusive convection in a micropolar ferromagnetic fluid. Appl. Math. Comp. 189, 1648-1661.

Sunil, P. Chand, P.K. Bharti and A. Mahajan (2008). Thermal convection in micropolar ferrofluid in the presence of rotation. J. Magn. Magn. Mater. 320, 316-324.

Sunil, P.K. Bharti and R.C. Sharma (2004). Thermosolutal convection in ferromagnetic fluid. Arch. Mech. 56, 117-135.

Venkatasubramanian, S. and P.N. Kaloni (1994). Effects of rotation on the thermoconvective instability of a horizontal layer of ferrofluids. Int. J. Eng. Sci. 32, 237-256. 